\title{
Pratiques
}

Linguistique, littérature, didactique

\section{Quels savoirs en matière de variations langagières susceptibles d'optimiser un enseignement du FLE}

\section{Françoise Favart}

\section{(2) OpenEdition}

12 Journals

Édition électronique

URL : http://journals.openedition.org/pratiques/1551

DOI : 10.4000/pratiques. 1551

ISSN : 2425-2042

Éditeur

Centre de recherche sur les médiations (CREM)

\section{Édition imprimée}

Date de publication : 15 juin 2010

Pagination : 179-196

\section{Référence électronique}

Françoise Favart, "Quels savoirs en matière de variations langagières susceptibles d'optimiser un enseignement du FLE », Pratiques [En ligne], 145-146 | 2010, mis en ligne le 15 juin 2010, consulté le 10 décembre 2020. URL : http://journals.openedition.org/pratiques/1551 ; DOI : https://doi.org/ $10.4000 /$ pratiques. 1551

\section{(c) Tous droits réservés}




\title{
Quels savoirs en matière de variations langagières susceptibles d'optimiser un enseignement du FLE
}

\author{
Françoise Favart \\ IUniversità degli Studi di Trento $(\mathrm{I})$ \\ et Laboratoire Modyco (Paris X)
}

\section{Introduction}

Composante fondamentale des langues, la variation linguistique trouve-t-elle sa place dans l'enseignement du Fle? Notre interrogation découle du constat que les apprenants de français langue étrangère de niveau avancé (B2/C1 du cadre européen commun de référence) auxquels nous sommes confrontée ont rarement été sensibilisés à la variation linguistique et en particulier à la variation diaphasique. La présence de la variation n'est en rien une particularité du français, mais le rôle qu'y joue actuellement la variation diaphasique justifie qu' on se demande s'il est encore fondé aujourd'hui d'enseigner le français comme une langue homogène. En outre, si nous admettons que la variation diaphasique est susceptible d'enrichir le bagage linguistique des apprenants, quelles stratégies mettre en œuvre pour les sensibiliser à une compétence qui, chez le locuteur natif, s'acquiert grâce à la multiplicité des situations de communication auxquelles il est confronté. Nous tenterons de répondre à ces questions en dressant tout d'abord un cadre théorique visant à préciser quelques notions liées à la variation et à la norme linguistiques. Nous présenterons ensuite le contexte didactique qui est à l'origine de nos observations. Enfin, nous conclurons en cherchant à proposer des pistes de réflexion visant à enrichir l'enseignement du Fle.

\section{La norme linguistique dans l'enseignement du Fle}

Admettre la présence de la variation langagière implique inévitablement la reconnaissance d'une norme linguistique. L'enseignement du Fle, comme celui des autres langues étrangères, passe en général par un premier geste qui consiste à éliminer de la langue que nous transmettons aux apprenants toute forme de variation. L'enseignement se construit alors à travers la transmission d'une norme. Ce terme étant hautement polysémique, nous passerons rapidement en revue les principales acceptions qui s'appliquent au domaine auquel nous nous intéressons ici. 


\subsection{La norme, les normes}

Le terme norme qu'A. Rey définit comme un «mot-piège » (1995:14) s'avère ambigu et malaisé à définir, car il se rattache à des notions multiples. Il n'est par ailleurs pas spécifique aux sciences du langage ou à la didactique des langues, puisque, de manière générale, il renvoie à une contrainte extérieure. On parle ainsi de normes sociales, de normes comportementales, etc. Le terme s'emploie alors au pluriel. Les multiples acceptions que nous connaissons aujourd'hui de ce mot sont, à des degrés divers, redevables de la conceptualisation qu'en a donné le sociologue É. Durkheim au début du $\mathrm{XX}^{\mathrm{e}}$ siècle. La norme linguistique trouve dans 1'enseignement, que ce soit du FLM ou du FLE, un terrain particulièrement fertile.

\subsubsection{Les approches binaires}

En linguistique, la norme peut être considérée comme un «sous-produit de la standardisation ${ }^{(1)}$ et implique l'idée que certaines façons de s'exprimer sont préférables à d'autres. Ce qui, par voie de conséquence, exclut toute forme de variation linguistique.

Depuis A. Rey (1972), on distingue la norme subjective de la norme objective. La première renvoie à l'idée de normé ou normatif et présuppose un usage valorisé. C'est la norme que l'on rencontre parfois écrite avec un $N$ majuscule et qui correspond à un "faisceau d'intentions subjectives » (Rey $1972: 5$ ). Elle s'inscrit dans une tradition puriste de la langue qui ne tient pas compte des nouveaux usages langagiers. Le purisme se caractérisant entre autres, par « la volonté de conserver à la langue une forme immuable - identifiable en fait à une élite de lettrés alors que tout l'appelle à changer [...] » (Yaguello 1988 : 96).

La seconde est observable et s'appuie sur l'idée de fréquence ou de tendance. Elle s'emploie parfois au pluriel ${ }^{(2)}$ et correspond à une situation objective et statistique. Elle renvoie à l'adjectif normal.

La norme subjective, qui se caractérise par une tendance à l'unification, impose aux locuteurs des jugements de valeurs structurés en un modèle hiérarchisé des usages. Si la norme subjective impose aux locuteurs une contrainte collective qui donne lieu à des jugements de valeur - quelle que soit la façon de parler de chacun -, il ne faut pas oublier qu'elle s'appuie en réalité sur la norme objective, sur les usages des locuteurs et sur les variations de ces usages. Nous nous permettons de rappeler que « seule une linguistique de la norme objective, de ses variations et de ses types, sous-jacents aux variations des usages, et une étude systématique des attitudes métalinguistiques dans une communauté utilisant le même système linguistique pourront fonder l'étude des normes subjectives, des jugements de valeur sur le langage » (Rey 1972: 16)

En effet, tout en avançant des motivations linguistiques ou culturelles, la véritable raison d'être de la norme subjective est sociale, au sens où elle « prend force de ce que, outre l'imposition par des institutions, elle est intériorisée par les locuteurs, même ceux qui ne la respectent pas » (Gadet 2003a : 19).

Par ailleurs, il est également fréquent de distinguer la norme descriptive de la norme prescriptive (Martinet 1974 : 6-7). La norme descriptive se rattache au

(1) Nous empruntons l'expression à Gadet (2003b : 108).

(2) Nous renvoyons entre autres aux définitions de A.-M. Houdebine-Gravaud (2002: 9-21). 
point de vue du linguiste et la norme prescriptive à celui du grammairien. Selon le point du vue du premier « différentes normes de réalisation d'une même langue coexistent nécessairement : le parler des paysans et celui des politiciens ne suivent pas les mêmes normes. La norme des puristes ou des grammairiens ne constitue qu'une d'entre elles » (Charaudeau et Maingueneau 2002 : 403).

La norme prescriptive choisit parmi les multiples usages d'une langue, ceux d'entre eux réputés comme corrects, conformes au standard; ceux qui respectent ce qu'il est courant de qualifier de «bon usage ». Grammairiens et puristes ont ainsi tendance à sélectionner de façon arbitraire des variantes qu'ils érigent en modèles uniques de langue, alors que les autres variantes sont proscrites. L'attitude des puristes consiste à sacraliser la norme et à proscrire tous les usages qu'elle ne reconnaît pas.

Aujourd'hui encore les normes subjective et prescriptive apparaissent comme prééminentes dans la didactique du Fle. Ainsi des énoncés pourtant courants et non marqués ${ }^{(3)}$ comme « demain j'suis pas là », « faut lui dire », « i partent en week-end », etc. sont rarement reconnus comme dignes d'être présentés aux apprenants. Ce type d'approche que d'aucuns considèrent comme indispensable - dans une première phase d'apprentissage pour certains, éternellement pour d'autres - s'avère dans tous les cas insuffisant, car il occulte une part importante des usages actuels de la langue française. En outre, il a l'inconvénient de ne pas prendre en compte les composantes communicationnelles tels que les genres de discours (Bakthine 1978), les locuteurs, le sujet énoncé, etc. Autant de paramètres qui interviennent pourtant dans tout échange linguistique.

\subsubsection{La norme pédagogique}

Les nombreux travaux dont nous disposons de nos jours sur la variabilité linguistique ont permis de dépasser le mythe de l'unicité et de l'uniformité de la langue. Toutefois, si les enseignants sont conscients qu'il n'est plus pensable de transmettre aux apprenants une image monolithique du français, ils se demandent aussi quel français enseigner ${ }^{(4)}$. Cette question est à l'origine de la réflexion d'A. Valdman (1998) qui souligne les difficultés auxquelles sont confrontés tous ceux qui tentent d'y répondre. Les problèmes sont d'autant plus importants que les méthodologies actuelles tendent à faire acquérir par les apprenants une maîtrise quasi native de la langue cible. Une solution serait alors d'établir « une norme neutre du français » (Idem : 177). Cette norme dite « pédagogique » s'appuie sur des critères d'ordres différents :

1. linguistiques : la norme pédagogique doit refléter le comportement observable des locuteurs dans la langue cible en tenant compte notamment des variantes linguistiques les plus fréquentes ou les plus caractéristiques des variétés diaphasiques et diastratiques ciblées,

2. épilinguistiques : les variantes proposées aux apprenants doivent coïncider avec l'idée que les locuteurs de la langue cible se font des productions linguistiques des différents groupes sociaux,

(3) Par non marqué, nous entendons des énoncés qui sont communs à la plupart des locuteurs et qui ne comportent pas de connotations sociales comme ce serait en revanche le cas pour un énoncé du type «comment qu'il s'appelle?».

(4) Cette question a notamment donné lieu à un colloque qui s'intéressait à la norme dans l'apprentissage (École polytechnique de Paris - septembre 2008). 
3. acquisitionnels : les variantes de la langue cible retenues doivent être en adéquation avec les compétences linguistiques des apprenants.

Ainsi la norme pédagogique a comme caractéristique première d'être observable, puisqu'elle prend en compte les variantes langagières les plus fréquentes des locuteurs de la langue étudiée. Ensuite, les variantes sélectionnées doivent correspondre aux attentes des membres de la communauté cible. En d'autres termes, elles doivent être en accord avec le parcours d'apprentissage, mais aussi avec les caractéristiques extra linguistiques des alloglottes. Enfin, sur le plan de l'acquisition, l'idée de progression domine. Elle incite à tenir compte des systèmes approximatifs par lesquels passe nécessairement l'acquisition de la version la plus approchée de la langue cible.

La norme pédagogique telle que la présente A. Valdman a l'avantage de prendre en compte des paramètres qui ne sont pas purement linguistiques. Par ailleurs, elle oblige les enseignants qui veulent s'en inspirer à s'interroger sur le degré de gravité des erreurs. On se demandera alors s'il est préférable de privilégier une erreur linguistique au prix d'une adaptation sociolinguistique et pragmatique ou s'il vaut mieux imposer coûte que coûte la correction linguistique au détriment de l'adaptation sociolinguistique.

\subsection{Les variations linguistiques}

Malgré l'impression d'unicité que donnent des appellatifs tels que la langue française ou encore le français, il n'existe pas de langue (naturelle) dépourvue de variations. Ces variations sont liées au temps, à l'espace, aux groupes sociaux, à l'individu et à la situation de communication. En outre, la variation peut toucher tous les domaines linguistiques : le phonique (par exemple : le pourcentage des $e$ muets prononcés ou élidés n'est pas le même à Lille qu'à Toulouse), le morphologique (Il va chez le boulanger / Il va au boulanger), le syntaxique (Où habitestu? / Tu habites où? / C'est où qu't'habites?), le lexical (un grain de beauté / un noevus) et le sémantique (la souris : «petit rongeur»/ « jeune femme, bonne amie » / « muscle, partie du gigot » / «boîtier connecté à un ordinateur »).

En sociolinguistique, le terme variété est habituellement utilisé pour désigner différentes façons de parler, alors que la variation se réfère plutôt aux phénomènes diversifiés en synchronie. Par ailleurs, il existe différentes manières de classer les variétés langagières. Ainsi après M.A.K. Halliday (1964), on peut distinguer la variation entre interlocuteurs, qui renvoie aux « différents individus selon les angles différents de diachronie, de localisation, et de position sociale » (Gadet 2007 : 23) de la variation intralocuteurs, qui se réfère au répertoire qu'adopte un même locuteur en fonction d'une situation de communication. On parlera alors de variation selon l'usage. Les différentes façons de s'exprimer d'un locuteur se distinguent elles aussi selon différents paramètres : temps, espace, caractéristiques sociales (telles que l'âge, le sexe, le niveau de scolarisation, etc.) et situations d'énonciation. Il sera alors respectivement question de variation de type diachronique (ou variation dans le temps), diatopique (ou variation dans 1'espace), diastratique (ou variation sociale) et diaphasique (ou variation situationnelle ou encore stylistique). Ces termes ont leur origine dans la romanistique allemande (Coseriu 1992 : 280) et ils ont l'avantage de se ranger dans l'ordre de la langue, contrairement à d'autres qui sont à cheval entre la référence à la langue et au monde.

Les variations diachronique, diatopique et diastratique relèvent de la variation 
selon l'usager, alors que la variation diaphasique concerne l'usage. C'est à cette dernière que nous nous intéressons dans cet article.

\subsubsection{La variation diaphasique}

La diversité terminologique, si fréquente en linguistique, fait que pour évoquer le domaine diaphasique ${ }^{(5)}$, on a recours à des termes tels que « niveau », « registre », «genre», « style» ou encore « variété stylistique ou fonctionnelle », etc. Le choix de l'un ou l'autre terme relève souvent de traditions nationales que nous n'entendons pas évoquer ici, si ce n'est pour signaler que «niveau » s'emploie exclusivement en français pour renvoyer à une opposition entre un usage formel et informel de la langue et comporte une connotation hiérarchisante. La notion de niveaux de langue - qui tend dernièrement à être remplacée par registre - est souvent appréciée dans l'enseignement du Fle. Cependant, elle a d'une part, l'inconvénient de tenir compte quasi exclusivement de l'ordre lexical ; de l'autre, elle véhicule l'idée « d'une langue de sens commun, selon laquelle il y aurait "différentes façons de dire la même chose" " (Gadet 2001 : 65).

La variation diaphasique est en réalité une notion plus vaste qui tient compte de l'expression changeante de l'individu en fonction des différentes situations de communication. Elle met en évidence le fait que certaines situations de communication exigent des formes d'expression qui s'imposent aux locuteurs et qui tiennent compte des protagonistes de l'échange, de la sphère d'activité et des objectifs qui animent l'échange, etc. Tout locuteur est ainsi capable de mettre en œuvre des compétences sociolinguistiques ${ }^{(6)}$, car il n'existe pas, comme le souligne W. Labov de « locuteur à style unique ». Toutefois, une distinction de taille s'opère entre le locuteur natif et l'apprenant de langue étrangère. Même s'ils sont peu nombreux, les travaux concernant l'acquisition du diaphasique chez les premiers mettent en évidence que cette maîtrise ne s'acquiert pas dans le cadre scolaire. Le locuteur natif dispose de références linguistiques variées ainsi que des références sociales externes qui sont associées et qui lui permettent de moduler son style en fonction des différentes situations d'énonciation. L'acquisition de ce type de variation relève selon W. Labov d'une phase avancée de ce qu'il appelle la « maîtrise totale d'une langue » ${ }^{(7)}$.

La réalité de l'apprenant de langue étrangère, chez qui les références sociales doivent être construites, est tout autre puisqu'il est soumis, dans la majorité des cas à un modèle de référence de la langue cible. Ce modèle correspond fréquemment à celui d'un français aseptisé, épuré, dépourvu de toute forme de variation. Dans la meilleure des hypothèses, l'enseignement passera par une distinction entre des usages oraux et des usages écrits de la langue.

(5) Définie comme variation stylistique par la tradition nord-américaine.

(6) La compétence sociolinguistique est définie par Lyster (1994:263) comme la capacité de reconnaître et de produire un discours socialement approprié en contexte. Ceci implique la capacité de savoir adapter son registre aux circonstances, à la situation, aux interlocuteurs, etc.

(7) Pour W. Labov la maîtrise de la variation diaphasique n'interviendrait pas avant l'âge de 14 ans. Nous précisons toutefois que d'autres travaux montrent que dès 3 ans, les enfants manifestent une sensibilité aux différents rôles sociaux (Slosberg Andersen 1990). 


\subsubsection{La primauté du diaphasique}

Toutes les langues naturelles connaissent des variations linguistiques, mais tous les types de variation ne jouissent pas de la même importance dans toutes les langues. En outre, la prééminence d'une variation peut évoluer avec l'histoire de la langue. Ainsi, la France, après avoir connu au XX $X^{\mathrm{e}}$ siècle une période où la variation diatopique était dominante, puis un primat du diastratique, liéà l'urbanisation et à l'industrialisation, voit se généraliser le diaphasique en corrélation avec une modification des normes comportementales en matière de relations sociales (Gadet 1998 : 62). On constate ainsi que la situation de la langue française diffère de celle des autres langues, notamment de l'italien où la domination est de l'ordre du diatopique (Berruto 1989) et de l'anglais où la variation dominante est de type diastratique.

\section{Le contexte didactique}

Al'encontre des approches méthodologiques qui font des apprenants d'éternels locuteurs de langue étrangère mais consciente des difficultés que comporte l'introduction de la variation - quelle que soit sa nature - en classe de Fle, nous proposons une réflexion qui repose essentiellement sur deux interrogations :

- quelles sont les raisons du décalage entre la langue que nous enseignons et la langue telle qu'elle est utilisée par les francophones?

- si la variation diaphasique est une propriété fondamentale de la langue française comment expliquer alors qu'elle ne trouve pas sa place dans l'enseignement du Fle?

Pour tenter d'apporter des éléments de réponse à ces deux questions nous partirons d'une analyse empirique de la situation que nous avons pu observer au cours d'une quinzaine d'années d'enseignement en milieu universitaire, dans le nord de 1'Italie. Cette observation a pour seul objectif de réfléchir à une réalité didactique qui, certes a beaucoup évolué depuis les années '80, mais qui pourrait, de notre point de vue, tirer profit des connaissances linguistiques dont nous disposons de nos jours.

\subsection{Les enseignants de Fle}

La prise en compte des deux principaux vecteurs de la langue que sont les enseignants et les manuels pédagogiques, nous porte à constater que trop souvent la langue que nous enseignons n'est qu'une version fortement appauvrie de la langue " authentique " ${ }^{(8)}$. Deux facteurs, au moins, peuvent expliquer ce constat.

Premièrement, les contraintes de temps auxquelles sont soumis les enseignants, le cadre formel dans lequel se déroule une leçon, ainsi que le contenu des programmes font - c'est pour le moins les objections avancées par les enseignants quand on leur suggère d'introduire de la variation dans leurs cours - que très souvent le parcours didactique proposé aux apprenants ne s'écarte guère du chemin tout tracé du manuel.

(8) Par langue authentique, nous entendons la langue telle qu'elle est utilisée par des locuteurs francophones dans différentes situations de communication. 
Deuxièmement, la difficulté qu'ont les enseignants - de part leur formation ${ }^{(9)}$, leur culture, leur position dans la classe ou encore le manque de recul par rapport à la méthodologie adoptée - à concevoir la variation linguistique comme une caractéristique fondamentale des langues. La variation figure dans l'enseignement du Fle, comme une scorie, un écart qu'il faut absolument éviter. On verra dans cette attitude les effets de ce que Milroy et Milroy définissent comme 1' " ideology of the standard» $(1985: 22)$. En d'autres termes, une tendance à considérer l'uniformité comme la situation idéale d'une langue.

Enfin, force est de constater que même s'il est prévu et recommandé par les programmes didactiques de développer les quatre compétences linguistiques que sont les compréhensions et les productions orales et écrites, le français écrit reste la composante de référence en matière d'enseignement. Nous n'entendons pas par là qu'aucune place n'est réservée à la pratique de l'oral en classe, mais nous voulons signifier que souvent l'oral est évalué et expliqué à travers le filtre de l'écrit et à travers les catégories qui lui sont propres, si bien que « l'oral s'oppose à l'écrit comme le mauvais français s'oppose au bon » (Blanche-Benveniste $2000: 35$ ).

\subsection{Les apprenants}

Si nous interrogeons les apprenants ${ }^{(10)}$, pour la plupart italophones, sur leur vision de la langue française, nous nous apercevons que le français de référence reste dans la plupart des cas, le français de France et de préférence le français parisien. Peu d'entre eux ont été sensibilisés à la variation linguistique ${ }^{(11)}$, même quand ils ont étudié le français au lycée pendant 5 ans. Cependant, tous ont appris qu'il existe une distinction entre la langue orale et la langue écrite, même si elle se présente sous la forme d'un amalgame entre l'oral familier voire populaire et l'écrit standard ou soutenu.

La possibilité de partir à l'étranger dans le cadre de leur cursus universitaire permet aux étudiants d'être confrontés à la langue telle qu'elle est réellement parlée. Toutefois, nous avons pu observer que si ces étudiants reviennent en Italie en ayant acquis une aisance à l'oral qu'ils n'avaient pas avant leur départ, ils sont rarement capables d'adapter leur style à la situation de communication. La plupart d'entre eux ont adopté des caractéristiques linguistiques propres à l'oral des étudiants qu'ils ont fréquentés, mais ils sont rarement conscients que ces marques caractéristiques d'un oral familier, courant chez les jeunes adultes, s'adaptent mal à une situation formelle tel qu'un examen, par exemple. Nous nous référons notamment à l'emploi de ouais, forme relâchée de oui. À leur retour d'un séjour de plusieurs mois dans une université francophone, les étudiants ont en général acquis cette forme de l'adverbe d'affirmation qui a remplacé la forme standard. Peu d'entre eux ont pris conscience que ces deux formes ne sont pas interchangeables. Nous illustrerons notre propos par le cas d'un étudiant de niveau B2, d'une faculté d'économie ayant effectué plusieurs périodes d'étude en France (Paris et Tours) et une période à l'université de Mons, en Belgique. Cet étudiant a constamment utili-

(9) Les enseignants que nous avons rencontrés ne sont pas tous de langue maternelle française et parmi ceux qui le sont tous n'ont pas conservé un contact direct et actualisé avec la langue qu'ils enseignent.

(10) Nous nous référons essentiellement à des apprenants de niveau avancé (B2-C1 du cadre européen commun de référence).

(11) Cet aspect de la question est amplement développé dans les travaux de C. Molinari (2008 et 2010). 
sé ouais comme l'équivalent de oui et il a manifesté le plus grand étonnement quand nous lui avons signalé que le ouais a un usage marqué et correspond à des situations de communication peu formelles et s'emploie essentiellement entre jeunes.

\subsection{Les manuels et grammaires pour le Fle}

Pour mesurer la place qui est réservée à la variation linguistique et plus précisément à la variation diaphasique dans les manuels et les grammaires utilisés en classes de français langue étrangère, nous avons sélectionné quatre phénomènes dont l'usage est corrélé à la variation diaphasique. Deux d'entre eux sont d'ordre morphologique, les deux autres relèvent du syntaxique. Parmi les phénomènes morphologiques, nous avons observé la présentation du pronom démonstratif ça et du pronom indéfini on. Sur le plan syntaxique, nous avons retenu la négation et l'interrogation.

Les trois grammaires auxquelles nous faisons références sont récentes. L'une d'entre elles est destinée aux apprenants de français langue étrangère de toutes origines confondues ${ }^{(12)}$. Les deux autres s'adressent à des italophones ${ }^{(13)}$. Pour chacun des faits de langue étudiés, nous donnons un extrait des explications que l'on peut lire dans les trois grammaires concernant l'usage de chaque phénomène. Quand cela nous semble apporter des éléments ultérieurs de réflexion, nous citons un exemple proposé dans ces mêmes ouvrages.

\section{Le pronom ça}

Ce pronom est généralement présenté comme l'équivalent oral de cela:

- Cela (ça à l'oral) est employé comme pronom sujet devant un verbe autre que être (G1 p. 52)

- Ça è una contrazione di cela e molto spesso, nella lingua parlata, lo sostituisce $^{(14)}$ (G2 p. 88). Exemple Ça fait combien?

- [...] à l'oral et dans un registre familier, les deux formes [ceci et cela] sont très souvent remplacées par une forme unique : ça (G3 p. 99)

\section{Le pronom on}

L’emploi de on est fréquemment associé à un registre familier :

- Nella lingua familiare, on puo' essere usato con il significato di nous (ma anche di $t u$ e vous $)^{(15)}$ (G2 p. 108)

- Dans certains usages stylistiques, on peut aussi remplacer nous. Cet emploi est considéré comme plus familier mais il est très courant [...] (G3 p. 81)

(12) Il s'agit de Y. Delatour, Nouvelle Grammaire du français (2004), dorénavant G1.

(13) Il s'agit de : D. Cornaviera, Entrez ...en grammaire (2007), dorénavant G2 et de F. Bidaud, Nouvelle grammaire du français pour italophones (2008), dorénavant G3.

(14) Nous traduisons : «Ça est une contraction de cela qui très souvent le remplace dans la langue parlée ».

(15) Nous traduisons : «Dans la langue familière, on peut être employé au sens de nous (mais aussi de $t u$ et vous)». 


\section{La négation}

Les trois grammaires que nous avons analysées signalent l'existence de la négation sans $n e$ :

- À l'oral, dans la langue familière, comme ne n'est pas tonique, on a tendance à le supprimer (G1 p. 190)

- Una frase negativa francese deve avere sempre due elementi negativi [...]. Solo nella lingua parlata familiare, è possibile sentire delle frasi costruite senza $n e^{(16)}(\mathrm{G} 2$ p. 229)

- Dans la langue parlée, on supprime souvent la particule ne (G3 p. 226)

\section{L'interrogation}

L'interrogation est un sujet complexe, nous observons ici l'interrogation totale de type direct, puisque c'est essentiellement à ce niveau que la variation linguistique est évoquée dans les manuels didactiques.

- Il y a trois structures interrogatives.

Intonation $\rightarrow$ la voix monte : langue familière.

Vous savez conduire? Oui, bien sûr!

Est-ce que... + forme affirmative : langue courante.

Inversion du sujet : langue soutenue (G1 p. 180)

- Esistono tre possibilità per formulare un'interrogazione: ${ }^{(17)}$

Con il tono della voce [...]. Allo scritto si mette un punto interrogativo. Questa forma viene usata nella lingua familiare.

Mettendo est-ce que all'inizio della frase, prima del verbo.

Con l'inversione del soggetto che viene messo dopo il verbo o dopo l'ausiliare se il tempo è composto [...]. Questa forma si usa in un linguaggio più formale $^{(18)}$ (G2 p. 234)

\section{- L'intonation}

Cette forme, caractéristique de l'usage oral, utilise la phrase affirmative en lui ajoutant une intonation montante [...]. À l'écrit, on ne l'utilise que pour la transcription des dialogues et on ne la distingue de la phrase assertive que par un point d'interrogation.

\section{L'inversion}

Elle consiste à déplacer le pronom sujet immédiatement après la partie conjuguée du verbe. L'inversion appartient à un registre de langue soutenu. Elle est relativement peu employée en français parlé parce qu'elle ne respecte pas l'ordre syntaxique traditionnel [...].

(16) Nous traduisons : «La phrase négative française doit toujours avoir deux éléments négatifs [...]. Dans la langue parlée familière uniquement, on peut entendre des phrases sans ne».

(17) Nous respectons les règles de ponctuation de l'italien, qui ne prévoit pas d'espace devant les « : ».

(18) Nous traduisons : «Il existe trois possibilités pour formuler l'interrogation : par intonation [...]. À l'écrit on met un point d'interrogation. Cette forme s'emploie dans la langue familière. En mettant la locution est-ce que au début de la phrase, avant le verbe. Par inversion du sujet qui est placé après le verbe ou après l'auxiliaire si le temps est composé. Cette forme s'utilise dans un langage plus formel ». 


\section{L'interrogation avec est-ce-que}

Cette forme, typique du français, est la plus employée parce qu'elle ne modifie pas l'ordre des mots de la phrase affirmative (G3 pp. 242-243)

Les explications grammaticales ci-dessus reflètent la présence d'un problème incontestable en didactique du français langue étrangère : la nécessité d'opérer des distinctions entre les différents usages d'un même phénomène linguistique.

Ces distinctions pourraient être considérées comme des tentatives pour introduire la variation linguistique dans des ouvrages destinés à décrire la langue française aux non natifs. Ainsi, les explications grammaticales énoncées plus haut reposent-elles essentiellement sur des distinctions variationnelles qui convoquent deux paramètres différents : d'une part le canal, de l'autre les registres de langue. En d'autres termes, il est question de faire entrer des faits de langue dans des catégories hermétiques bien définies : la langue orale $v s$ la langue écrite ou le registre familier $v s$ le registre soutenu, etc.

Les grammaires que nous avons analysées proposent une première distinction entre l'oral et l'écrit, c'est notamment le cas pour l'emploi du pronom ça tel qu'il est présenté dans les grammaires G1 et G2. C'est également sur une distinction entre la langue parlée et la langue écrite que se base la présence ou l'absence du ne de négation en $\mathrm{G} 3$.

Le choix d'une variété peut en outre être présenté comme relevant d'un registre de langue spécifique (essentiellement familier ou soutenu). On relève cette distinction en G3, pour le pronom ça. Il en est de même pour le phénomène linguistique le plus fréquemment évoqué en matière de registre dans l'enseignement du Fle : l'interrogation ${ }^{(19)}$. L'interrogation par intonation est présentée comme caractéristique d'un registre familier, sauf dans la grammaire G3 où cette forme est décrite comme une tournure de l'oral ou servant à représenter des traits d'oralité à l'écrit. Quant à l'inversion, les trois grammaires signalent cet usage comme soutenu ou formel. L'interrogation par est-ce-que en revanche se rattache à une question de registre uniquement pour G1. Cette tournure est associée pour G3 à une question de fréquence.

Enfin, un troisième type de distinction apparaît dans le cas de la négation sans ne. Elle convoque à la fois le canal et le registre. Ainsi, selon G1 et G2, ce type de négation est propre de la langue orale dont le registre est familier. C'est également sur ces deux paramètres que repose l'emploi de ça en G1.

\section{Le canal et le registre permettent-ils de sensibiliser au diaphasique?}

Ces tentatives de classement ont le mérite de sensibiliser les apprenants à des usages distincts de certains faits de langue. Toutefois, ils évoquent une idée d'équivalence entre les différentes formes selon que l'on a affaire au canal écrit ou au canal oral. Il est ainsi courant, dans la représentation collective, de considérer l'oral comme une version simplifiée, du point de vue syntaxique et lexical, de l'écrit. De plus, les distinctions qui reposent uniquement sur une opposition au niveau du canal (oral / écrit) ont l'inconvénient d'ignorer les paramètres extralinguistiques (locuteurs en présence, sujet traité, cadre le l'échange communicationnel, etc.) qui interviennent dans toute situation de communication.

(19) Sur ce point nous renvoyons à A. Valdman (1998). 
En outre, si le classement de la variation linguistique en différents registres $(f a-$ milier, courant ou soutenu ) donne l'impression de proposer des distinctions plus fines, nous nous demandons toutefois si une telle hiérarchisation des phénomènes linguistiques est un moyen suffisamment efficace pour sensibiliser l'apprenant à la variation linguistique. Ce type d'approche perpétue une fois de plus l'idée d'équivalences linguistiques et de catégories fermées dans lesquelles insérer différents faits de langue.

Ensuite, les notions de familier, courant, soutenu auxquelles on a fréquemment recours pour «classer» des faits de langue sont-elles aussi nettement définies que l'on pourrait le croire? Si tel est le cas, quels seraient alors les paramètres qui permettent de considérer un registre comme familier? Il semble par exemple communément admis que le vouvoiement d'un interlocuteur ne puisse être considéré que comme une marque de distance qui exclurait toute familiarité. Pourtant, si nous nous référons aux extraits cités précédemment, nous observons que l'interrogation par intonation, qui est présentée comme caractéristique d'un registre familier est illustrée en G1 par l'exemple suivant : Vous savez conduire? Oui, bien sûr ! Si cet exemple a pour but de reproduire un extrait de situation de communication tel qu'on pourrait l'entendre dans la langue in vivo, nous ne pouvons nous empêcher de nous demander quels sont, dans cet énoncé, les éléments qui permettent de déduire qu'il y a familiarité entre les interlocuteurs.

Le registre familier est également convoqué dans le choix de le négation sans ne. G2 illustre son affirmation par deux exemples : Je veux pas y aller et Je sais pas (p. 229). Si ces deux énoncés peuvent très bien correspondre à des situations de familiarité, nous ne sommes pas convaincue que le corrélat soit vrai. En d'autres termes, nous considérons que Je veux pas y aller et Je sais pas pourraient très bien s'entendre dans des situations de communication tout à fait courantes, dans lesquelles n'apparaîtrait aucun indice de familiarité. Les nombreux travaux sur l'oral ne laissent désormais aucune place à l'ambiguïté : la négation sans ne est considérée comme un phénomène tout à fait courant. C. Blanche-Benveniste fait remarquer qu'il y a environ $95 \%$ d'absence de $n e$ dans les conversations, quels que soient les locuteurs (Blanche-Benveniste 2000 : 39). Dans la langue orale authentique, la négation sans ne ne coïncide ni avec le statut social des locuteurs, ni avec une situation de communication spécifique.

L'exemple suivant repose quant à lui sur la distinction entre l'oral et l'écrit. On peut lire en G2 que souvent ça remplace cela à 1'oral. Une affirmation qu'illustre la phrase ci-après : Ça fait combien ? Doit-on alors imaginer que cet exemple transposé à l'écrit deviendrait : Cela fait combien? Cette construction est, de notre point de vue, fort peu envisageable. Ne devrions-nous pas plutôt concevoir l'emploi de ça, tout comme celui des autres phénomènes évoqués, en relation au type d'énoncé, au genre de discours et à la situation de communication dans laquelle il a été produit?

Les exemples auxquels nous avons fait référence, confortent, nous semble-t-il, une impression selon laquelle il existe un amalgame entre les deux plans distincts de la langue que sont l'oral et l'écrit et les registres linguistiques : soutenu, standard, familier, populaire, etc. Il est ainsi fréquent que la langue orale soit associée à un registre familier ou populaire, alors que l'écrit est considéré comme soutenu ou courant. Cette association n'a aucune raison d'être puisque l'oral aussi bien que l'écrit disposent de différents registres. On a ainsi affaire à une sur-légitimité de l'écrit au dépend de l'oral et à une non prise en compte de la diversité des situations et des genres de discours. 
Il résulte alors de nos observations que les distinctions qui reposent sur le canal ou sur le registre donnent une vision limitée de la variation diaphasique et sont insuffisantes pour réduire l'écart entre la langue que nous enseignons et la langue telle qu'elle est utilisée par les locuteurs natifs.

Nous tenons cependant à préciser que parmi les trois grammaires que nous avons analysées, G3 est incontestablement celle qui manifeste la plus grande sensibilité à la variation linguistique.

\section{L'apprenant pluristyle, une illusion?}

À ce stade de notre réflexion, nous retenons que d'une part la variation diaphasique est dominante dans le français d'aujourd'hui. De l'autre que, malgré les tentatives proposées par différentes grammaires, cette variation ne semble guère être prise en considération dans la didactique du Fle. La variation est dans tous les cas considérée comme un élément qui viendrait s'ajouter à la langue normée. Peu importe si la place de certains phénomènes linguistiques est massive dans le français d'aujourd'hui ? Peu importe si le français que nous transmettons diffère des nombreux français qui se parlent?

\subsection{Ouvrir le Fle aux autres domaines de recherche}

Les réflexions et observations que nous avons proposées ci-dessus confirment le décalage qui existe entre la langue enseignée en Fle et la langue telle que 1'utilise les locuteurs francophones. La sensibilisation à la variation diaphasique pourrait contribuer au rapprochement de ces deux entités. Plusieurs pistes mériteraient d'être explorées pour optimiser l'enseignement du Fle en matière de variation. Nous pensons entre autres aux nombreux travaux sur 1'oral (Blanche-Benveniste), à ceux des interactionnistes dans le domaine de la didactique, mais nous nous limiterons ici à l'apport des travaux des romanistes allemands $\mathrm{P}$. Koch et W. Esterreicher. L'approche de ces deux linguistes s'écarte de l'opposition traditionnelle basée exclusivement sur le canal pour mettre en avant-plan la situation de communication avec les différents éléments qui lui sont associés : 1'interaction, le lieu, le temps, etc.

\subsection{Repenser l'oral et l'écrit dans l'enseignement du Fle}

Les deux auteurs proposent une distinction stricte entre les aspects médiaux et les aspects conceptionnels de 1'oral et de l'écrit. En cela, ils suivent les propositions du romaniste allemand L. Söll (1974) qui met en évidence la différence entre l'aspect médial (code phonique vs code graphique) et conceptionnel d'un énoncé. Il applique à ce dernier les termes de langue parlée et de langue écrite. Le médial et le conceptionnel permettent ainsi quatre combinaisons : "parlé phonique ", "parlé graphique », «écrit phonique » et « écrit graphique ». Chez certains linguistes, comme W. Chafe (1982), les termes informel et formel ${ }^{(20)}$ remplaceront parlé et écrit. Cependant cette opposition ne couvre qu'une partie de l'espace conception-

(20) W. Chafe distingue quatre «styles » de langue : « informal spoken language, formal spoken language, informal written language, formal written language » (Chafe 1982: 36). Nous traduisons par : « langue parlée informelle, langue parlée formelle, langue écrite informelle, langue écrite formelle ». 
nel. P. Koch et W. Esterreicher vont aller plus loin en adoptant les termes d'immédiat et de distance. Ces termes ne constituent pas une dichotomie comme c'était le cas pour phonique et graphique, mais correspondent aux deux extrémités d'un continuum communicatif et " englobent la totalité des paramètres conceptionnels » (Koch et Esterreicher 2001 : 586). Ce continuum communicatif repose, en dernière analyse, sur des données anthropologiques, à la base de toute communication humaine. L'oral et l'écrit devraient ainsi être entendus comme des phénomènes particuliers de l'immédiateté et de la distance. Ce type de distinction pourrait, nous semble-t-il, enrichir l'opposition entre oral et écrit, qui comme nous l'avons montré ne peut répondre de manière satisfaisante à l'enseignement du Fle.

Le tableau ci-dessous illustre les principaux paramètres communicatifs mis en évidence par les deux auteurs.

\begin{tabular}{|c|c|}
\hline Immédiat & Distance \\
\hline Communication privée & Communication publique \\
\hline Interlocuteur intime & Interlocuteur inconnu \\
\hline Émotionnalité forte & Émotionnalité faible \\
\hline Ancrage actionnel et situationnel & Détachement actionnel et situationnel \\
\hline Ancrage référentiel dans la situation & Détachement référentiel dans la situation \\
\hline Co-présence spatio-temporelle & Séparation spatio-temporelle \\
\hline Coopération communicative intense & Coopération communicative minime \\
\hline Dialogue & Monologue \\
\hline Communication spontanée & Communication préparée \\
\hline Liberté thématique & Fixation thématique \\
\hline$[\ldots]$ & {$[\ldots]$} \\
\hline
\end{tabular}

Paramètres communicatifs de l'immédiat et de la distance (Koch et CEsterreicher 2001 : 586).

Il apparaît dans ce tableau non exhaustif, qu'il n'est pas tant question de traits typiques de 1'oral ou de l'écrit, mais plutôt de situations, qui selon les contextes d'activités, incitent à privilégier un type de production plutôt qu'un autre. Ce cadre réserve une place privilégiée au locuteur et met l'accent sur la co-présence, l'interaction et l'engagement de ce dernier. Il est évident que pour certains types de discours, le recouvrement entre immédiat et oral ou entre distance et écrit est partiel, et que seuls certains paramètres peuvent être appliqués. Nous pensons par exemple à une conversation téléphonique, qui bien que relevant de l'immédiat ne comporte en général pas la co-présence spatiale. Toutefois, les paramètres présentés dans le tableau pourraient être utilisés dans l'enseignement du Fle pour aider les enseignants à affiner des distinctions peu satisfaisantes en matière de sensibilisation à la variation diaphasique.

\subsection{Pistes didactiques}

L'approche que nous proposons ici n'ayant pas été exploitée, à notre connaissance, dans le domaine du Fle, nous suggérons trois pistes didactiques qui ont pour but de faire réfléchir les apprenants au fonctionnement énonciatif et discursif des faits de langue évoqués précédemment. 


\subsubsection{Exercice à partir d'un article de presse}

Après avoir lu l'article de presse intitulé Maurice ou les portes de l'Inde et expliqué les difficultés grammaticales et lexicales, nous revenons sur différents passages pour essayer de comprendre quels sont les paramètres communicatifs qui interviennent dans le choix du pronom on:

À Maurice, la conduite est sportive. On circule à gauche, en slalomant entre les tas de bois sur les bas-côtés et les bus, cacochymes et hauts en couleur [...]. Détail pittoresque, on ne voit quasiment que des casques rouges. Pour aller d'une plage à l'autre, on profite des bateaux-taxis.

Le marché de Poste de Flacq est plus confidentiel. On n'y voit pas un touriste, seulement des Mauriciens.

Plus au sud, un autre lieu étonnant, le parc Vanille, dans la commune de Rivière des Anguilles. On peut y admirer des crocodiles de toutes tailles [...], un troupeau de tortues terrestres, centenaires pourcertaines et pesant près de $100 \mathrm{~kg}$. On est même autorisés à poser le temps d'une photo sur le dos de ces pauvres bêtes qui semblent tout droit sorties de Jurassic Park. ${ }^{(21)}$ (Maurice ou les portes de l'Inde, tiré de http://madame.lefigaro.fr/loisirs-et-voyages/carnets-de-voyages/33-maurice-oules-portes-de-linde).

En nous basant sur le tableau de P. Koch et W. Esterreicher (2001), nous demandons aux étudiants de préciser la valeur du pronom on et son fonctionnement dans l'article étudié. Ce pronom étant fréquemment présenté dans les grammaires comme l'équivalent familier de nous (éventuellement d'autres pronoms sujets), nous invitons les étudiants à le remplacer par ce qui est censé être un équivalent. Il apparaît alors que dans les extraits proposés, on ne peut être considéré, sur le plan énonciatif, comme l'équivalent de nous. On voit bien, en effet que on circule à gauchen'est pas l'équivalent de nous circulons à gauche. On observe en revanche que on a une fonction englobante, qui réunit à la fois le référent (les Mauriciens), parfois l'énonciateur (le journaliste) et le destinataire (le lecteur ou touriste potentiel). On remarque aussi que malgré la distance physique entre le journaliste et ses lecteurs, le pronom on crée un rapprochement. De plus, une phrase comme on ne voit que des casques rouges laisse entendre que le journaliste s'est rendu sur l'île et confère de l'authenticité au texte.

Si nous nous référons aux paramètres communicatifs présents dans le tableau cité plus haut, nous constatons que les interlocuteurs ne sont pas intimes, mais que on permet de réduire la distance entre l'énonciateur et le destinataire du message. On observe également que l'ancrage référentiel dans la situation de communication est fort et ceci même si le lecteur n'est pas à Maurice. Ces différents éléments permettent de comprendre que l'emploi du on ne peut être envisagé ni comme uniquement propre à l'oral, ni comme l'équivalent familier d'un autre pronom sujet.

Des exercices semblables peuvent être proposés pour d'autres faits de langue communément attribués à l'oral. Nous pensons notamment au démonstratif ça.

\subsubsection{Exercice à partir de transcriptions}

S'il est assez simple aujourd'hui de trouver des documents audio en français, il s'avère moins aisé de se procurer des enregistrements provenant de situations de

(21) C'est nous qui soulignons. 
vie quotidienne. Pour cette raison, nous nous appuyons sur les transcriptions de C. Blanche-Benveniste (2005) et de V. Traverso (1996). Les extraits qui suivent nous ont permis de travailler sur la négation sans $n e$.

(1) L- vous avez pas vu Françoise Dolto l'aut'fois

F- moi j'ai vu r'gardé euh j'sais pas ça d'vait durer une heure non. (Traverso $1996: 134)$

(2) $\mathrm{P}$ - demain tu viens manger chez moi à midi

L- j'sais pas tu m'as pas invitée

P- ben ouais j't'ai pas eue au téléphone. (Traverso 1996 : 135)

(3) L2- on peut pas vous pouvez pas y échapper. (Blanche-Benveniste et alii 2005 : 231)

(4) L2- bon alors donc vous vous pouvez pas vous vous grillager chez sur le mur. L1- ah non non non on peut pas grillager. (Blanche-Benveniste et alii $2005: 234$ )

Pour chacun des exemples ci-dessus, les étudiants ont essayé de mettre en évidence les paramètres communicatifs propres à chaque situation de communication. Il apparaît ainsi que dans les quatre exemples l'ancrage actionnel et situationnel, ainsi que l'ancrage référentiel sont des constantes, tout comme la co-présence spatio-temporelle. Nous n'avons pas tenu compte des paramètres tels que le type de communication (privée ou publique) ou encore le type d'énoncé (dialogue $v s$ monologue) puisque, dans tous les cas, nous avons affaire à des conversations en vis-à-vis. Par ailleurs, on observe dans l'exemple (4) et peut-être aussi dans l'exemple (3) que la négation sans nen'est pas conditionnée par un rapport intime entre les interlocuteurs ni par un haut degré de familiarité.

\subsubsection{Exercice à partir d'une source audio}

Nous avons travaillé sur un extrait d'interview à J. Semprun (Les Matins de France culture du 24 février 2010) pour observer le fonctionnement de l'interrogation et en particulier de l'interrogation par intonation, régulièrement présentée comme propre au registre familier. Pour ce faire nous avons demandé aux étudiants de relever les formes interrogatives présentes dans un passage d'environ 4 minutes :

Journaliste - Tout est exact à peu près, à gros traits ?

- Est-ce que c'est comme ça que vous souhaitez vous, vous présenter?

- Est-ce que vous seriez le premier à revendiquer sur une carte d'identité Jorge Semprun, Européen?

- Vous n'avez pas de patrie?

- Apatride vous irait bien?

- Vous prenez le meilleur de chacun, c'est ça ?

- Les abrazos, les accolades espagnoles, à Paris, ne vous manquent pas?

- C'est comment Don Quichotte en allemand?

Semprun - Je suis indécis sur la demande « Vous êtes espagnol ou français ?»

Le relevé des formes interrogatives met en évidence que l'interrogation par inversion est totalement absente de cet extrait. Quant à l'interrogation par est-ce que, elle est employée à deux reprises par le journaliste. Toutes les autres phrases interrogatives passent par l'intonation. Un fois de plus, nous constatons qu' on ne 
peut limiter ce type d'interrogation à un registre familier. On observe en revanche que l'interrogation par intonation est associée à des paramètres communicatifs qui relèvent à la fois de l'immédiat et de la distance ou qui se situent dans le continuum entre ces deux extrémités. Nous remarquons notamment :

- que les interlocuteurs en présence ne sont pas intimes, puisqu'ils se vouvoient ;

- que la communication ne peut être considérée comme privée, puisqu'elle a été retransmise en direct à la radio ;

- qu'il existe une coopération communicative intense entre les interlocuteurs ;

- qu'il est question d'un dialogue, etc.

Il nous semble ainsi pouvoir conclure que le recours fréquent à l'interrogation par intonation est étroitement lié aux caractéristiques énonciatives et au genre discursif de chaque texte. Il en va de même pour les autres faits de langue que nous avons analysés.

\section{Pour conclure}

Si nous admettons avec Hymes (1991) que l'interprétation globale de l'acte de communication est assurée par des compétences de communication et non pas par les seules compétences linguistiques, l'image du français que nous donnons aux apprenants de langue étrangère mérite d'être enrichie. Deux éléments, nous semble-t-il, pourraient nous aider à optimiser notre enseignement. Tout d'abord, il serait intéressant de considérer l'enseignement de la langue étrangère comme une partie intégrante d'un enseignement de compétences communicationnelles. Ensuite, nous devrions nous munir d'instruments qui nous permettraient de développer ces compétences tout en respectant les contraintes spécifiques à la didactique. Sur le plan théorique, les paramètres proposés par P. Koch et W. Esterreicher nous paraissent des références extrêmement utiles. En effet, grâce à l'introduction de l'aspect « conceptionnel », elles brisent les catégories hermétiques d'oral et d'écrit pour mettre en avant le continuum communicatif. Une vision qui d'ordinaire n'est pas prise en compte dans la didactique du Fle. Décider d'intégrer l'idée de continuum dans les cours de Fle impose que l'enseignant réserve une place importante aux éléments extra linguistiques qui interviennent dans tout acte de communication. Il n'est certes pas aisé d'y arriver si on tient compte que l'enseignement frontal renvoie à des schémas communicationnels rigides. En outre, l'utilisation du tableau présenté ci-dessus nécessite une connaissance profonde et actualisée de la langue française. Nous ne reviendrons pas sur la diversité que nous avons observée sur le plan de la formation des enseignants de Fle et sur le rapport que chacun entretient avec la langue française, autant d'éléments qui peuvent constituer un obstacle à l'introduction du diaphasique dans les classes de français langue étrangère. On voit ainsi toutes les difficultés que comporte le recours à la variation en didactique. Toutefois, 1'emploi massif de documents authentiques variés sur lesquels nous demanderions aux apprenants de réfléchir en tenant compte des paramètres de $\mathrm{P}$. Koch et W. Esterreicher, pourrait être un premier pas dans une tentative de sensibilisation des allophones à la variation diaphasique. Un bon moyen peut-être pour réduire la distance entre langue enseignée et langue pratiquée par les locuteurs natifs. 


\section{Bibliographie}

BAKHTINe, M. (1978) : Esthétique et théorie du roman, traduction française de D. Olivier, Paris, Gallimard [édition originale 1975].

Berruto, G. (1989) : Sociolinguistica dell'italiano contemporaneo, Rome, La Nuova Italia Scientifica.

BlanCHE-BENVENiste, C. (2000) : Approche de la langue parlée en français, Paris, Ophrys [ $1^{\text {re }}$ édition 1997].

BLANCHE-BENVENISTE, C. et alii (2005) : Le français parlé, études grammaticales, Paris, Éditions du CNRS [1 ${ }^{\text {re }}$ édition 1990].

CHAFE, W. (1982) : «Integration and Involvement in Speaking, Writing, and Oral Literature ", in Tannen D. Editor, Spoken and Written Language : Exploring Orality and Literacy, Norwood, NJ, Ablex P.C., pp. 35-53.

Charaudeau, P., Maingueneau, D., sous la direction de (2002) : Dictionnaire d'analyse du discours, Paris, Seuil.

COSERIU, E. (1992) : Einführung in die allgemeine Sprachwissenschaft, Tübingen, Francke Verlag [ $1^{\text {re }}$ édition 1962].

GADET, F. (1998) : « Cette dimension de la variation que l'on ne sait nommer », Sociolinguistica, $\mathrm{n}^{\circ} 12$, pp. 63-68.

— (2001) : «Enseigner le style », Le français dans le monde, numéro spécial, pp. 53-71.

- (2003a): La variation sociale en français, Paris, Ophrys, coll. L'essentiel.

- (2003b) : «La variation : le français dans l'espace social, régional et international », in Yaguello M. (sous la direction de), Le grand livre de la langue française, Paris, Éditions du Seuil.

- (2007) : La variation sociale en français, nouvelle édition revue et augmentée, Paris, Ophrys, coll. L'essentiel.

HALLIDAY, M.A.K. (1964) : "The user and uses of language » repris in Fishman J. (sous la direction de), Readings in the Sociology of Language, La Haye-Paris, Mouton [1968].

HoudeBINE-GRAVAUD, A.-M., sous la direction de (2002) : L'imaginaire linguistique, Paris, L'Harmattan, coll. Langue et Parole.

HYMES, D. (1991) : Vers la compétence de communication, Paris, Hatier/Didier.

KOCH, P., ESTERREICHER, W. (2001) : «Langage parlé et langage écrit », Lexikon der romanistischen Linguistik, tome 1, Tübingen, Max Niemeyer Verlag, pp. 584-627.

LABOV, W. (1976) : Sociolinguistique, traduction française de Alain Kihm, Paris, Les Éditions de Minuit, coll. Le Sens Commun [édition originale 1972].

LYSTER, R. (1994) : "The effect of functional-analytic teaching on aspects of French immersion students' sociolinguistic competence », Applied Linguistics, $\mathrm{n}^{\circ} 15,263-287$.

Martinet, A. (1974) : Éléments de linguistique générale, Paris, Armand Colin [1 ${ }^{\text {re }}$ édition 1967].

Milroy J., MilRoy, L. (1985) : Authority in Language, Londres, Routledge \& Kegan Paul.

MOLINARI, C. (2008) : «L'enseignement du FLE face au défi de la variation », in Alao G. et alii, Grandes et petites langues. Pour une didactique du plurilinguisme et du pluriculturalisme, Berne, Peter Lang, pp. 57-68.

- (2010): «Normes linguistiques et normes culturelles dans l'apprentissage 
du FLE : un parcours d'ouverture à la variation francophone ", in Bertrand O.et Schaffner I., Quel français enseigner? La question de la norme dans l'enseignement/apprentissage, Paris, Les Éditions de l'École Polytechnique, 2010, pp. 101-114.

REY, A. (1972) : «Usages, jugements et prescriptions linguistiques », Langue française, $\mathrm{n}^{\circ} 16$, pp. 4-28.

- (1995) : « Norme et dictionnaire ou 1'arbitraire a toujours tort», Le Français aujourd'hui, Vol. 148, pp. 9-14.

Slosberg Andersen, E. (1990) : Speaking with Srtyle. The Sociolinguistic skills of Children, Londres-New York, Routledge.

SÖLL, L., HAUSMANN, F.J. (1974) : Gesprochenes und geschriebenes Französisch, Berlin, Grundlagen der Romanistik.

TRAVERSO, V. (1996) : La conversation familière. Analyse pragmatique des interactions, Lyon, Presses Universitaires de Lyon.

VALDMAN, A. (1998) : La notion de norme pédagogique dans l'enseignement du Fle, in Bilger M., Van den Eyden K. et Gadet F., Analyse linguistique et approches de l'oral. Recueil d'études offert en hommage à C. Blanche-Benveniste, Leuven/Paris, Peeters, pp. 177-188.

Y AGuello, M. (1988) : Catalogue des idées reçues sur la langue, Paris, Éditions du Seuil.

\section{Grammaires étudiées}

BIDAUD, F. (2008) : Nouvelle grammaire du français pour italophones, Novara, UTET.

CORNAVIERA, D. (2007) : Entrez ...en grammaire, Turin, Loescher.

Delatour, Y et alii, (2004) : Nouvelle Grammaire du français, Paris, Hachette. 